\title{
Broadband X-ray edge-enhancement imaging of a boron fibre on lithium fluoride thin film detector
}

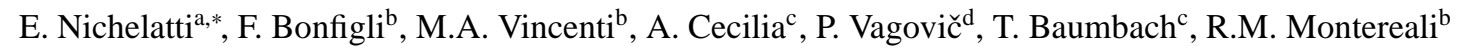 \\ ${ }^{a}$ ENEA C.R. Casaccia, Fusion and Technologies for Safety and Security Dept., Photonics Micro- and Nano-structures Laboratory, \\ FSN-TECFIS-MNF, Via Anguillarese 301, 00123 S. Maria di Galeria, Rome, Italy \\ ${ }^{b}$ ENEA C.R. Frascati, Fusion and Technologies for Safety and Security Dept., Photonics Micro- and Nano-structures Laboratory, \\ FSN-TECFIS-MNF, Via E. Fermi 45, 00044 Frascati, Rome, Italy \\ ${ }^{c}$ Institute for Photon Science and Synchrotron Radiation, Karlsruhe Institute of Technology (KIT), Karlsruhe, Germany \\ ${ }^{d}$ Center for Free-Electron Laser Science (CFEL), Deutsches Elektronen-Synchrotron (DESY), Notkestrasse 85. Bldg. 99, 22607 Hamburg, \\ Germany
}

\begin{abstract}
The white beam $(\sim 6-80 \mathrm{keV})$ available at the TopoTomo X-ray beamline of the ANKA synchrotron facility (KIT, Karlsruhe, Germany) was used to perform edge-enhancement imaging tests on lithium fluoride radiation detectors. The diffracted X-ray image of a microscopic boron fibre, consisting of tungsten wire wrapped by boron cladding, was projected onto lithium fluoride thin films placed at several distances, from contact to $1 \mathrm{~m}$. X-ray photons cause the local formation of primary and aggregate colour centres in lithium fluoride; these latter, once illuminated under blue light, luminesce forming visible-light patterns—acquired by a confocal laser scanning microscope- that reproduce the intensity of the X-ray diffracted images. The tests demonstrated the excellent performances of lithium fluoride films as radiation detectors at the investigated photon energies. The experimental results are here discussed and compared with those calculated with a model that takes into account all the processes that concern image formation, storing and readout.
\end{abstract}

Keywords: Radiation detectors, X-rays, X-ray edge-enhancement imaging, Lithium fluoride, Colour centres, Photoluminescence

\section{Introduction}

Dielectric materials containing point defects [1] are successfully utilized in several fields, including radiation detection [2]. For instance, colour centres (CC's) [3] in lithium fluoride (LiF) are well known for their application in tuneable solid-state lasers [4], dosimeters [5], and miniaturized light-emitting devices [6]. Certain types of CC's in LiF are optically active, stable at room temperature, and possess broad absorption and emission bands in the visible [7]. Among them, $\mathrm{F}_{2}$ and $\mathrm{F}_{3}^{+}$aggregate centres, consisting of two electrons bound to two and three closely spaced fluorine vacancies, respectively, emit in the red $\left(\mathrm{F}_{2}\right)$ and green $\left(\mathrm{F}_{3}^{+}\right)$portions of the spectrum and have almost overlapping absorption bands peaked at the wavelength of $\sim 450 \mathrm{~nm}$ (M band) [7]. For this reason, $\mathrm{F}_{2}$ and $\mathrm{F}_{3}^{+}$centres can be simultaneously excited with a single-wavelength optical pump in the blue.

Because of their high-penetration properties, X-rays are widely used to analyze the internal structure of objects, including biological samples. Absorption contrast is perhaps the most elementary and intuitive technique utilized to record radiographies; this is possible when samples present details whose absorption properties are clearly distinguishable (contrasted) at the operating X-ray wavelengths. On the other hand, for high-energy X-rays, the absorption coefficient of most materials is relatively small so that they behave as almost pure phase objects. The lack of absorption contrast can be compensated by adding phase information to the recorded image [8]. A possible approach to that is edge-enhancement imaging, also known as in-line phase-contrast imaging $[8,9]$.

${ }^{*}$ Corresponding author

Email address: enrico.nichelatti@enea.it (E. Nichelatti) 
Edge-enhancement imaging is a technique-utilized with spatially coherent X-rays-based on wave free propagation that allows enhancing visibility of sharp spatial details of samples by detection of the transmitted electromagnetic wavefront after it has freely propagated in the near field [10-16]. When an X-ray beam is transmitted through a sample that consists of parts with different refractive indices and absorption coefficients, its wavefront locally experiences distinct amplitude and phase changes. While, in case of a sample consisting of solely absorbing materials at the probing wavelengths, the borders among the sample parts can be visualized even on a detector, sensible to electromagnetic intensity, placed at zero distance (absorption contrast), differences in phase are not directly visible in a setup where sample and detector are in contact because they induce no direct intensity change. Fresnel diffraction [17] comes in helpful in this case, because it is a known fact that at increasing distances from the sample more and more spaced Fresnel fringes appear along lines separating wavefront areas that bring distinct phase information, thus making them clearly visible provided that the wavefront intensity is recorded by a suitable high-resolution detector. As a matter of fact, interference fringes in Fresnel diffraction are proportional to the second spatial derivative (Laplacian) of the phase of the wavefront $[11,16]$, so that they appear where abrupt phase changes take place, like those due to borders among materials with different refractive indices. Applicability of edge-enhancement imaging is strongly related not only to the characteristic of the utilized X-ray source, especially as far as its transverse coherence properties are concerned, but also to those of the detector, which should be able to spatially resolve the Fresnel fringes.

High-resolution X-ray imaging can be accomplished with a variety of methods [18]; however, all of them are limited by the resolution and/or the dynamic range of the most common radiation detectors. Solid-state LiF detectors, based on radiation sensitivity of the material and optical readout of photoluminescence (PL) from $\mathrm{F}_{2}$ and $\mathrm{F}_{3}^{+} \mathrm{CC}^{\prime}$, can overcome the above-mentioned limitations so that they become potentially suitable for the characterization of micro and nanostructures as well as biological samples. High spatial resolution across a large field of view, wide dynamic range, and versatility make them very convenient as recording plates for both X-ray absorption-contrast [19$23]$ and phase-contrast [24, 25] imaging. LiF imaging radiation detectors were successfully used by using several X-ray sources, such as synchrotrons [25-27], laser plasma sources [20, 21, 28, 29], capillary discharge lasers [30], table X-ray tubes [22, 31].

Suitableness of LiF, either in bulk or thin film form, as edge-enhancement imaging detector for white beam synchrotron X-rays was recently tested at the ANKA light source of the KIT synchrotron facility in Karlsruhe, Germany [25]. The test consisted of transmitting the white beam spectrum through an Xradia test pattern, and exposing LiF-based detectors, placed at a distance of $17.5 \mathrm{~cm}$, to the transmitted beam. Optically-active CC's were created, in this way, in $\mathrm{LiF}$, with a transversal distribution reproducing the diffracted shadow of the test pattern. With no further treatment or special handling, the stored CC distributions were detected and acquired as bi-dimensional maps of visible PL in a fluorescence microscope, by illuminating the exposed LiF samples with blue light in order to optically pump $\mathrm{F}_{2}$ and $\mathrm{F}_{3}^{+} \mathrm{CC}$ 's [25].

In the present paper, the results of a systematic experiment are reported and discussed. With a selected specimen, consisting of a boron fibre (BF) [32], LiF thin-film radiation detectors were tested in an X-ray edge-enhancement imaging setup in the same beamline of [25], that is by using a white X-ray beam whose energy spectrum ranges approximately from 6 to $80 \mathrm{keV}$. In the literature other papers are available where BF's are dealt with as far as imaging with coherent X-rays is concerned [33-38]; the main novelty here is the use of luminescent LiF film detectors instead of more traditional ones, such as scintillators and CCD arrays or cameras. Furthermore, with respect to a recent paper reporting a similar test at the same beamline [25], in the present study a good portion of the sample- the B cladding - is an essentially pure phase object with refractive index gradient along one axis, while in [25] the sample was mostly an amplitude object. Another difference is that here the LiF detectors are placed at various distances from the specimen, ranging from complete contact to $1 \mathrm{~m}$, to test their suitableness to both absorption-contrast (concerning the $\mathrm{W}$ wire) and phase-contrast imaging. As in [25], the CC distributions generated in LiF are later observed as bidimensional PL maps under the blue light of a confocal laser scanning microscope (CLSM); one-dimensional cross sections of the detected maps are here compared with those calculated with a theoretical model. The results confirm the good performances of LiF films as imaging detectors for broadband X-ray beams, even when used with samples having important phase-only components.

The paper is organized as follows. The experimental part is reported in section 2, while the theoretical model is explained in section 3. In section 4 a comparison between experimental and simulated PL maps is reported and discussed. Section 5 closes the paper with conclusions. 


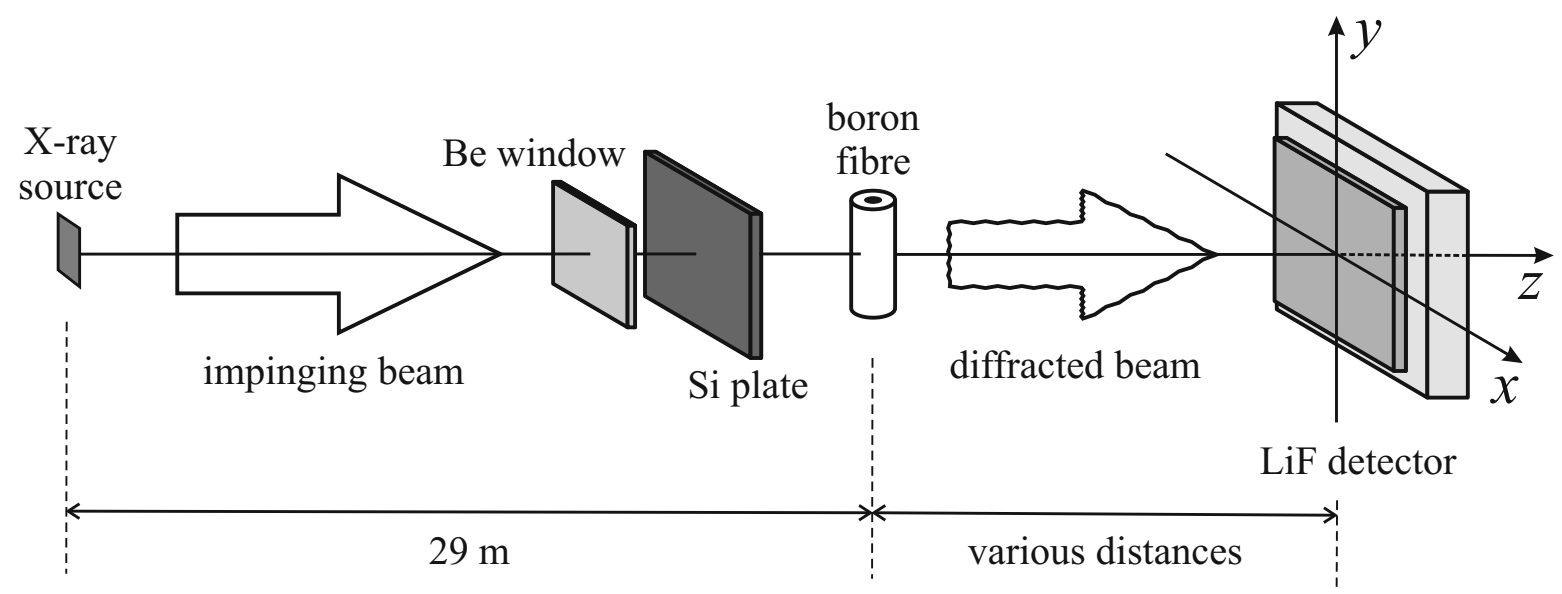

Figure 1: Simplified scheme of the irradiation setup in the X-ray edge-enhancement imaging experiment on LiF film detector at the TopoTomo beamline of the ANKA light source (Institute for Synchrotron Radiation, Karlsruhe Institute of Technology KIT, Germany).

\section{Experimental}

The irradiations were performed at the TopoTomo beamline of the ANKA light source (Institute for Synchrotron Radiation, Karlsruhe Institute of Technology KIT, Germany). The ANKA synchrotron facility is operated with an electron energy of $2.5 \mathrm{GeV}$. The TopoTomo beamline can work either in 'white beam' (poly-energetic) or in monochromatic mode; the available white-beam energy spectrum ranges approximately from 6 to $80 \mathrm{keV}$. Other specifications are available elsewhere [25] and online at the ANKA website [39].

For the X-ray imaging experiment here reported, the TopoTomo beamline was operated in white-beam mode. After an in-vacuum propagation of $27.7 \mathrm{~m}$, a $250 \mu \mathrm{m}$ thin vacuum-sealing berillium (Be) window allowed the passage of the beam into air, where a $2 \mathrm{~mm}$ thick silicon ( $\mathrm{Si}$ ) plate was placed before the sample in order to cut the beam low-energies and thus minimize the absorbed dose. The distance of the BF from the X-ray source was $29 \mathrm{~m}$. After interacting with the $\mathrm{BF}$, the beam wavefront freely propagated to the $\mathrm{LiF}$ film detector, that was placed at various distances - five sampled distances from contact to $1 \mathrm{~m}$ - from the BF itself. A scheme of the experimental setup is shown in figure 1. The total (i.e., spectrally integrated) intensity of the white beam onto the sample was estimated to be about $1 \mathrm{~W} / \mathrm{mm}^{2}$.

\subsection{Sample}

Boron fibres - typically obtained by depositing amorphous elemental boron (B) onto the surface of a fine metallic wire substrate - present very interesting mechanical and physical properties [32] so that they are used in the aerospace industry and for the realization of strong and lightweight composite materials. In our experiment we used one of such fibres, precisely a $\mathrm{W}$ cylindrical wire ( $15 \mu \mathrm{m}$ diameter) embedded in a cylindrical B cladding (100 $\mu \mathrm{m}$ total diameter); in figure 2 a drawing of the BF cross section is shown. The reason for selecting such a sample in an X-ray edgeenhancement imaging test is that, at the energies of the white beam, the $\mathrm{W}$ core is quite absorbing, while the $\mathrm{B}$ cladding is almost a pure phase object. This fact, illustrated in figures 3 and 4 , allows for testing the edge-enhancing capabilities of the imaging technique and, in parallel, the usability of the LiF film as high-resolution detector.

Regarding figure 3, the plotted optical constants of $\mathrm{B}$ and $\mathrm{W}$ up to $100 \mathrm{keV}$ are available from the refractive-index database of the IMD extension of XOP software, which is downloadable from the European Synchrotron (ESRF) website [40]. On the other hand, the optical constants of $\mathrm{LiF}$ were found only up to $30 \mathrm{keV}$, and they are available at the Center for X-Ray Optics (CXRO) website [41]; the LiF values of $\delta$ and $\beta$ from $30 \mathrm{keV}$ to $100 \mathrm{keV}$ shown in figure 3 were linearly extrapolated in semi-logarithmic scale.

\subsection{LiF film detectors}

Two identical polycrystalline LiF films, of thickness $1.7 \mu \mathrm{m}$, were deposited by thermal evaporation on glass substrates, which were kept at a constant temperature of $300^{\circ} \mathrm{C}$, under a vacuum pressure of about $10^{-4} \mathrm{~Pa}$ in a 


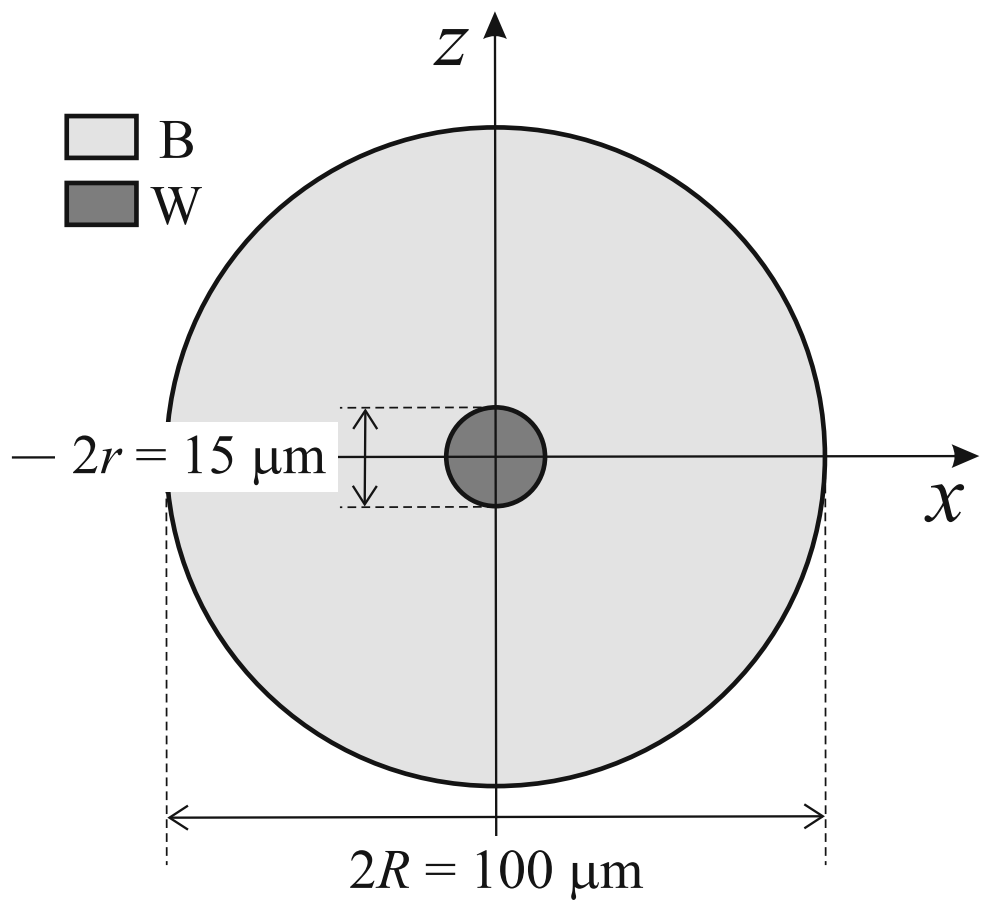

Figure 2: Cross-section of the boron fibre used for the X-ray edge-enhancement imaging experiment.

dedicated evaporation facility at ENEA C.R. Frascati. The starting material was LiF microcrystalline powder (Merck Suprapur, $99.99 \%$ pure), heated at about $800^{\circ} \mathrm{C}$ in an open tantalum crucible. The final thickness and the evaporation rate were fixed and in situ monitored by an INFICON quartz oscillator, placed close to the substrate holder.

\subsection{Readout of the photoluminescence maps}

The X-ray radiographies stored in the LiF film detectors were optically read by a CLSM (Nikon Eclipse 80i-C1) operating in fluorescence mode with a $40 \times$ objective of numerical aperture NA $=0.75$. During the optical readout, the $\mathrm{LiF}$ films were illuminated from above by the $457.9 \mathrm{~nm}$ line of a continuous-wave argon laser; the spectrally integrated PL signals of $\mathrm{F}_{2}$ and $\mathrm{F}_{3}^{+} \mathrm{CC}$ 's were simultaneously detected by two different photomultipliers. The detection bit depth was 12 bit. Coloured glass filters in front of each photomultiplier were used to spectrally separate the red and green PL signals [42].

Figure 5 shows the red-channel PL maps that were detected for the five BF-LiF test distances. All of them correspond to an exposition time of $60 \mathrm{~s}$ to X-rays. The five $200 \mu \mathrm{m} \times 250 \mu \mathrm{m}$ photos in figure 5 were cropped from the full instrumental field of view of the CLSM, corresponding to a $318 \mu \mathrm{m} \times 318 \mu \mathrm{m}$ square sampled in a $1024 \times 1024$ pixel matrix. The first map, figure 5(a), was obtained by placing the BF sample directly in contact with the LiF detector and was recorded on the first of the two available films. The other four PL maps, figure 5(b-e), were obtained by progressively increasing the $\mathrm{BF}-\mathrm{LiF}$ distance and were all recorded in distinct areas on the second available $\mathrm{LiF}$ film. It can be clearly noticed how the B cladding, not visible in figure 5(a) because of its transparency to X-rays, becomes more and more distinguishable at increasing distances due to the phase-contrast edge enhancement caused by Fresnel diffraction of the X-ray wavefront.

\section{Theoretical model}

The theoretical model that we utilized to tentatively reproduce the experimental results of section 2.3 is a sequential evaluation of (a) beam-sample interaction, (b) wave free propagation from sample to detector, (c) generation of CC's in the detector, (d) emission of PL from the CC's. Transverse coherence properties of the X-ray beam and finite spatial 

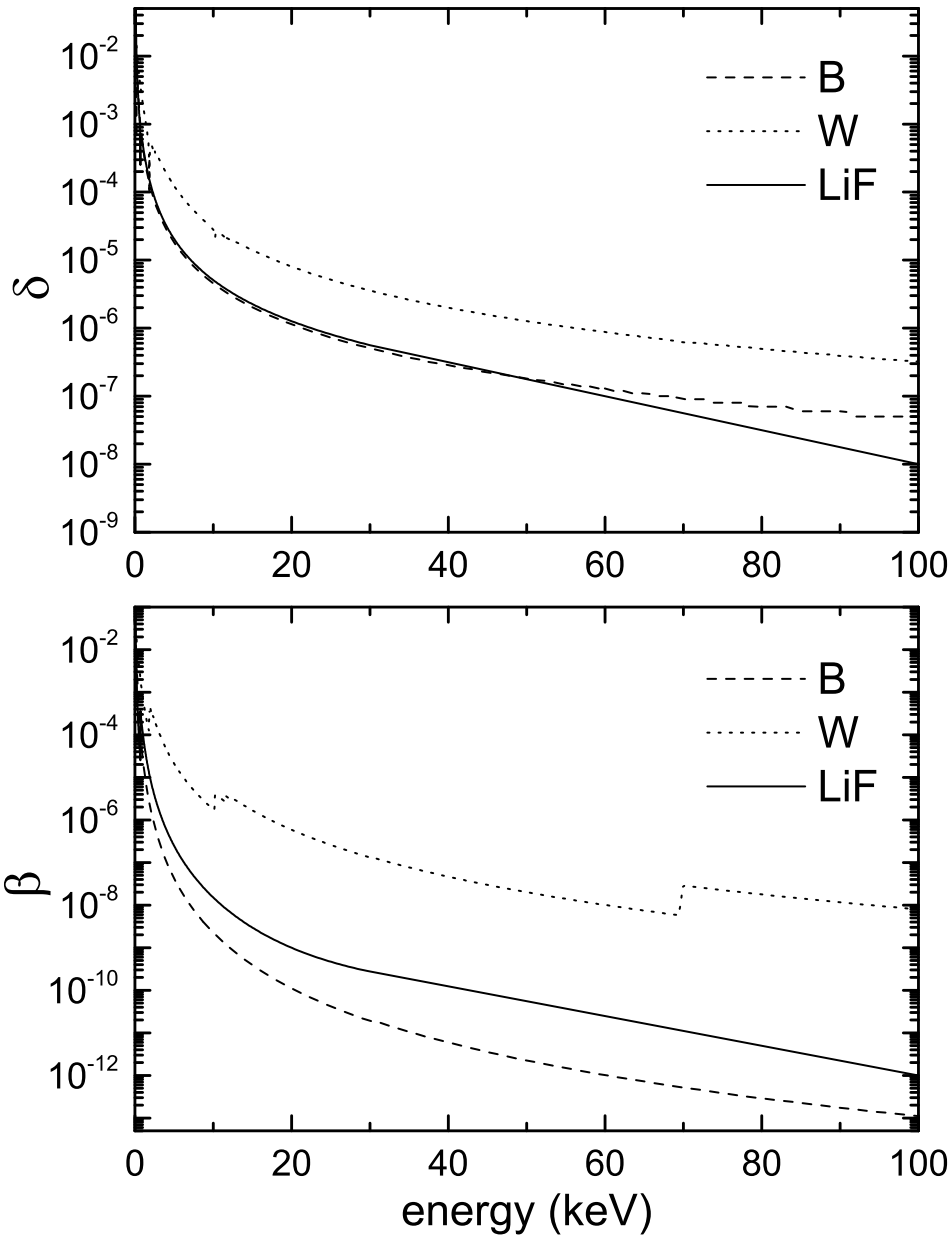

Figure 3: Complex refractive indices of the materials used in the simulation of the X-ray edge-enhancement imaging experiment: $\mathrm{B}$ and $\mathrm{W}$ (boron fibre), and $\mathrm{LiF}$ (thin film detector). The top figure shows the $\delta$ component of the real part of the indices; the bottom figure shows the imaginary part $\beta$ of them (the complex refractive index is defined as $n=1-\delta-\mathrm{i} \beta$ ). 


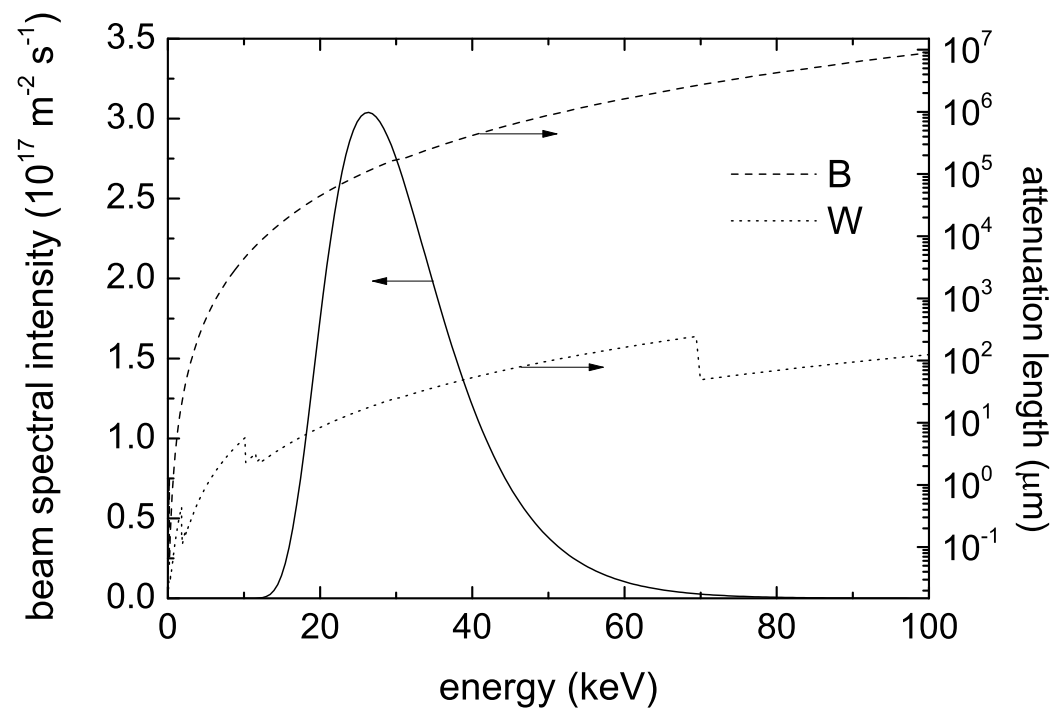

Figure 4: Attenuation lengths of B and W (right scale) as calculated from tabulated data of the optical constants (see figure 3). The spectral intensity of the X-ray beam (left scale) is also plotted; in correspondence of its peak at $26.4 \mathrm{keV}$ the attenuation lengths are $\sim 11 \mathrm{~cm}$ for B and $\sim 17 \mu \mathrm{m}$ for W.
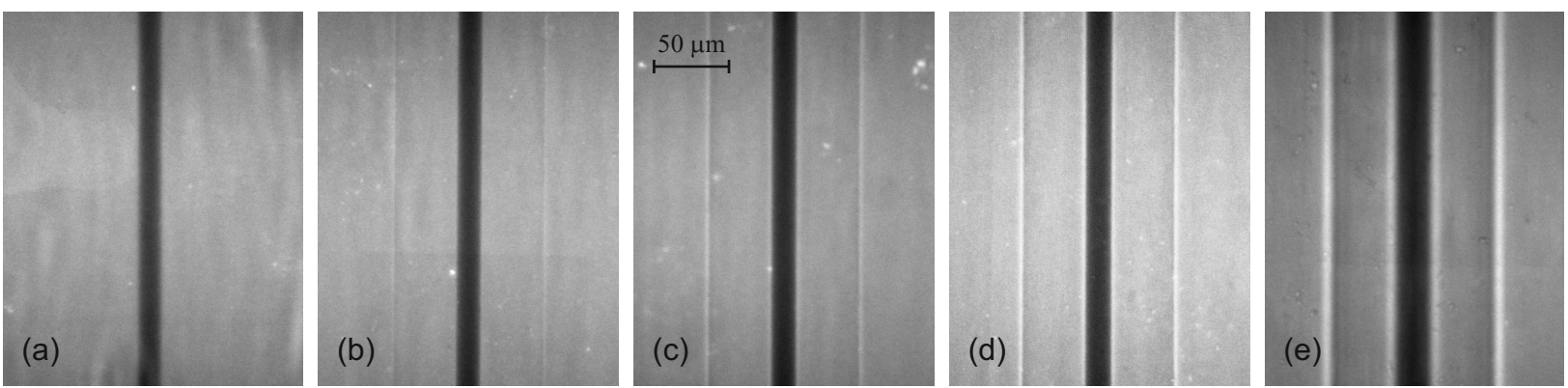

Figure 5: Confocal-microscope screenshots $(40 \times$ objective, numerical aperture NA $=0.75$, fluorescence mode, red channel) of the detected PL maps reproducing the diffracted images of the $\mathrm{BF}$ that were stored in LiF films as $\mathrm{CC}$ distributions. From left to right, the five photos correspond to increasing BF-LiF distances: (a) $0 \mathrm{~mm}$ (contact); (b) $18 \mathrm{~mm}$; (c) $50 \mathrm{~mm}$; (d) $100 \mathrm{~mm}$; (e) $1000 \mathrm{~mm}$. 
resolution of the PL readout microscope are taken into account. As far as free propagation of the X-ray wavefront from the sample to the detector is concerned, it is a simple application of Fresnel's diffraction formula at each wavelength component of the spectrum. This is a well known step in simulating phase-contrast imaging [13] and a fundamental one since it causes the appearance of Fresnel fringes, thus giving rise to the actual edge-enhancement phenomenon; here we approached it from a plane-wave spectrum $[43,44]$ viewpoint for numerical convenience.

\subsection{Interaction of the $X$-ray beam with the sample}

The starting assumption is that the X-ray wavefront impinging onto the sample can be considered plane at each wavelength. The fact that it is originated from a finite-size (rather than point) source influences its transversalcoherence properties, but this fact will be addressed later on: for now, a fully coherent plane wave is being assumed.

Let us model the X-ray beam transmitted through the Be window and the Si plate with a continuous superposition, $\int_{0}^{\infty} \psi(x, y, z ; E) \mathrm{d} E$, of monoenergetic plane waves, each described by the wavefunction

$$
\psi(x, y, z ; E) \equiv \sqrt{I(E)} \exp \left(-\mathrm{i} \frac{2 \pi}{\lambda} z\right)
$$

where the adopted Cartesian reference system $O x y z$ is visible in figure 1. Here, $E$ and $\lambda$ are the photon energy and wavelength, respectively, mutually related by $\lambda E=h c$, where $h$ is Planck's constant and $c$ the velocity of light in vacuum. The intensity distribution $I(E)$ is the energy spectrum shown in figure 4 (left scale). The interaction of the wave with the BF is accounted for by means of a complex-amplitude transmittance function,

$$
t(x, y ; \lambda)=\exp [-\mathrm{i} \phi(x, y ; \lambda)]
$$

where the argument $\phi(x, y ; \lambda)$, deducible from the geometry of the cross-section drawn in figure 2 , is defined by

$$
\phi(x, y ; \lambda)=\left\{\begin{aligned}
\frac{4 \pi}{\lambda}\left[R+\left(n_{\mathrm{B}}-1\right) \sqrt{R^{2}-x^{2}}\right. & \\
\left.+\left(n_{\mathrm{W}}-n_{\mathrm{B}}\right) \sqrt{r^{2}-x^{2}}\right], & |x| \leq r \\
\frac{4 \pi}{\lambda}\left[R+\left(n_{\mathrm{B}}-1\right) \sqrt{R^{2}-x^{2}}\right], & r<|x| \leq R \\
\frac{4 \pi}{\lambda} R, & |x|>R
\end{aligned}\right.
$$

As shown in figure 2, $r$ and $R$ are the radius, respectively, of the internal $\mathrm{W}$ core and of the $\mathrm{B}$ cladding, while $n_{\mathrm{W}}$ and $n_{\mathrm{B}}$ are their complex refractive indices - see figure 3 . Note how the argument $\phi(x, y ; \lambda)$ is generally complex-valued, so that the complex-amplitude transmittance of equation (1) causes both a phase shift and an amplitude attenuation of the crossing wave. Note also the invariance of $\phi$ along $y$, due to the chosen orientation of the BF along that axis, so that the explicit dependence on $y$ of $\phi, t$ and $\psi$ can be safely dropped.

Right after the BF, let us set it to be $z=z_{0}$, the wavefunction is

$$
\psi\left(x, z_{0} ; E\right)=\sqrt{I(E)} \exp \left(-\mathrm{i} \frac{2 \pi}{\lambda} z_{0}\right) \exp [-\mathrm{i} \phi(x ; \lambda)] .
$$

The wave is no more plane because, for what said above, it has acquired an explicit dependence on the transversal coordinate $x$ due to its interaction with the sample. For this reason, its propagation in the free space to the $\mathrm{LiF}$ film detector needs to be calculated with Fresnel's diffraction formula.

\subsection{Propagation of the $X$-ray beam to the LiF detector}

The dimensions and symmetry of the problem allow considering the paraxial approximation of the scalar Helmholtz equation [43] for the propagation of the wave from the BF to the LiF detector. By adopting the plane-wave spectrum formalism $[43,44]$, the wavefunction at the LiF film detector plane $z=z_{\mathrm{d}}$ can be written as the Fourier integral

$$
\psi\left(x, z_{\mathrm{d}} ; E\right)=\int_{-\infty}^{+\infty} \Psi\left(\xi, z_{0} ; E\right) \exp \left(\mathrm{i} \pi \lambda D \xi^{2}\right) \exp (-\mathrm{i} 2 \pi \xi x) \mathrm{d} \xi
$$

equivalent to Fresnel's diffraction formula, where $\Psi\left(\xi, z_{0} ; E\right)$ is the Fourier transform of $\psi\left(x, z_{0} ; E\right)$ along $x, \xi$ is the spatial frequency conjugated to $x, D \equiv z_{\mathrm{d}}-z_{0}$ is the BF-LiF distance, an unessential constant phase term in front 
of the integral has been omitted, and we recall that $\lambda$ is function of $E$ because of $\lambda E=h c$. This Fourier integral is conveniently tackled by means of Fast Fourier Transform (FFT) algorithms [45] at each sampled energy $E$ of the white-beam spectrum. We accomplished this task in Matlab [46] within an ad hoc program that included all the stages of the simulation and provided all the results shown in this paper.

\subsection{Influence of transverse coherence}

So far, the fact that the X-ray beam is emitted from a source of finite size (200 $\mu \mathrm{m} \times 800 \mu \mathrm{m}$ [39]) has been neglected, thus assuming as perfectly coherent the wave associated to the beam. However, the wavefront emitted by a source that is not infinitely small is not ideal as far as spatial coherence is concerned. A way to include partial coherence in the calculations consists of convolving the fully-coherent intensity with a properly scaled version of the source distribution function (SDF) [47]. Here, for simplicity, a Gaussian SDF is utilised,

$$
\operatorname{SDF}_{0}(x)=\exp \left(-\frac{x^{2}}{\sigma_{\mathrm{s}}^{2}}\right),
$$

whose full-width at half-maximum (FWHM) is $1.67 \sigma_{\mathrm{s}}$. Its scaled version on the detector plane depends on the BF-LiF distance $D$, and is calculated as [47]

$$
\operatorname{SDF}(x)=\operatorname{SDF}_{0}\left(\frac{z_{\mathrm{s}}}{D} x\right)
$$

$z_{\mathrm{s}}$ being the distance between source and BF, $z_{\mathrm{s}}=29 \mathrm{~m}$ in the present paper. A FWHM value of $200 \mu \mathrm{m}$ gave the best results among the ones tested in the range $0-1000 \mu \mathrm{m}$, as far as the reproduction of experimental PL profiles, shown later in this paper, is concerned.

On the surface of the LiF film detector, the spectral intensity of the incoming diffracted X-ray beam, momentarily assumed as fully coherent, is

$$
I\left(x, z_{\mathrm{d}} ; E\right)=\left|\psi\left(x, z_{\mathrm{d}} ; E\right)\right|^{2} .
$$

The mathematical convolution of $I\left(x, z_{\mathrm{d}} ; E\right)$ with $\operatorname{SDF}(x)$

$$
\hat{I}\left(x, z_{\mathrm{d}} ; E\right)=I\left(x, z_{\mathrm{d}} ; E\right) * \operatorname{SDF}(x),
$$

gives the actual spectral intensity of the incoming X-ray beam for the case of finite-size source.

\subsection{Density of radiation-induced colour centres}

Reflection of X-rays at the air-LiF interface can be safely neglected at the energies considered in this paper: the reflection coefficient is smaller than $10^{-11}$ for photon energies larger than $6 \mathrm{keV}$ and up to $100 \mathrm{keV}$. Therefore, considering only transmitted X-rays, the probability density of a photon of energy $E$ to be absorbed at a certain depth $z_{\mathrm{LiF}}$ in $\mathrm{LiF}$ after crossing the $\left[0, z_{\mathrm{LiF}}\right]$ material slice is easily derived from Lambert-Beer's law [48] and amounts to

$$
p\left(z_{\mathrm{LiF}}, E\right)=\alpha(E) \exp \left[-\alpha(E) z_{\mathrm{LiF}}\right]
$$

where $\alpha(E) \equiv \frac{4 \pi E}{h c} \beta(E)$ is the absorption coefficient of $\operatorname{LiF}$ (a plot of $\beta$ vs. $E$ is shown in figure 3 ). It is readily recognized that the energy density that is deposited per unit time (rate of deposited-energy density) at the depth $z_{\mathrm{LiF}}$ is

$$
\dot{\varepsilon}\left(x, z_{\mathrm{LiF}}\right)=\int_{0}^{\infty} p\left(z_{\mathrm{LiF}}, E\right) \hat{I}\left(x, z_{\mathrm{d}} ; E\right) \mathrm{d} E .
$$

Assuming for simplicity the absorbed dose to be small enough that the volume density $N$ of generated CC's (of any kind) is well below saturation-so that it can be considered to be directly proportional to the deposited-energy density - it ensues that, after the X-irradiation, the density of CC's in the LiF film is expected to be

$$
N\left(x, z_{\mathrm{LiF}}\right)=\mu \dot{\varepsilon}\left(x, z_{\mathrm{LiF}}\right),
$$

where $\mu$ is a suitable coefficient that depends on the host material, the considered kind of CC's, and is proportional to the irradiation time. Equation (12) gives the density distribution of CC's that are created in the radiation-exposed LiF film detector. 


\subsection{Colour-centre photoluminescence map}

Considering optically-active CC's in LiF, in particular $\mathrm{F}_{2}$ and $\mathrm{F}_{3}^{+}$aggregate centres, one recognizes from the above discussion that a visible PL map can be extracted from the irradiated detector under suitable conditions. These include the possibility of recording such a map at an optical microscope, when the LiF detector is illuminated with blue light tuned to the absorption band ( $\mathrm{M}$ band, peak wavelength of $\sim 450 \mathrm{~nm}$ ) of the $\mathrm{F}_{2}$ and $\mathrm{F}_{3}^{+} \mathrm{CC}$ 's.

In the simplest approximation, assuming the volume density of CC's to be that described by equation (12), the PL intensity radiated from any chosen kind of active $\mathrm{CC}$ can be estimated to be

$$
I_{\mathrm{PL}}(x)=\int_{0}^{h} q\left(z_{\mathrm{LiF}}\right) N\left(x, z_{\mathrm{LiF}}\right) \mathrm{d} z_{\mathrm{LiF}} .
$$

Here, $h$ is the film thickness, while $q\left(z_{\mathrm{LiF}}\right)$ is a suitable function that accounts for interference effects, both of the pumping light and of the PL itself, due to the film boundaries, plus other factors, including the quantum efficiency of the process. Neglecting, for simplicity, such interference effects, one can write

$$
I_{\mathrm{PL}}(x) \simeq \bar{q} \int_{0}^{h} N\left(x, z_{\mathrm{LiF}}\right) \mathrm{d} z_{\mathrm{LiF}},
$$

$\bar{q}$ being an average value of $q\left(z_{\mathrm{LiF}}\right)$.

Before proceeding with the comparison between experimental data and simulations, it is worth noting that the use of a microscope objective for the observation of a PL map implies that the finest map details get blurred by the finite spatial resolution $\sigma_{\mathrm{m}}$ of the microscope optics. This fact can be taken into account by convolving $I_{\mathrm{PL}}(x)$ with a suitable point spread function (PSF) [49]. Assuming for simplicity a PSF of Gaussian shape,

$$
\operatorname{PSF}(x)=\exp \left(-\frac{x^{2}}{\sigma_{\mathrm{m}}^{2}}\right),
$$

satisfactory results were obtained by setting its half-width at half-maximum (HWHM) to be equal to twice the Rayleigh's diffraction limit [17], that is by setting $0.83 \sigma_{\mathrm{m}}=1.22 \lambda_{\mathrm{ob}} / \mathrm{NA}$, where $\lambda_{\mathrm{ob}}$ is the observation wavelength and NA the numerical aperture of the microscope objective. In the present case, the observation of the red channel suggests choosing for $\lambda_{\mathrm{ob}}$ the peak emission wavelength of $F_{2}$ centres, $\lambda_{\mathrm{ob}} \simeq 650 \mathrm{~nm}$, while the numerical aperture of the $40 \times$ objective utilized in the measurements was NA $=0.75$. With these values, one gets $\sigma_{\mathrm{m}} \simeq 1.3 \mu \mathrm{m}$. An estimation of the PL map that is actually detected by the microscope is therefore

$$
\hat{I}_{\mathrm{PL}}(x)=I_{\mathrm{PL}}(x) * \operatorname{PSF}(x),
$$

which is the quantity that is calculated and compared with experimental data in section 4 .

\section{Comparison between measurements and simulations}

The results of the previous sections are here utilized to mutually compare experimental and theoretically calculated data. In particular, scans along a horizontal line of the measured PL maps shown in figure 5 are here compared with simulations obtained from equation (16).

Linear profiles of the PL maps of figure 5 were scanned orthogonally across the fibre direction, that is, along the $x$-axis (see figure 1), by using pixel-intensity detection software. The resulting experimental PL profiles are shown in the five plots of figure 6 (left side), starting from the zero-distance one at the top of the figure, and with increasing distances going from the top to the bottom. Side by side, the corresponding theoretical PL profiles calculated by means of equation (16) are also displayed in figure 6.

One can notice how these theoretical profiles reproduce fairly well the experimental ones for all the five sampled distances. Some differences can be however noticed. Besides the unavoidable presence of noise in the measured profiles, the most noticeable difference regards the PL intensity corresponding to that part of the X-ray beam that interacted with the $\mathrm{W}$ core: the calculated one is lower than the measured one in the spatial range $-7.5 \mu \mathrm{m} \leq x \leq$ $7.5 \mu \mathrm{m}$, which corresponds to the $\mathrm{W}$ core diameter of the BF. Indeed, as shown in figure 6 , the theoretical intensity transmittance at $x=0$, depending on the chosen BF-LiF distance, is $\sim 0.3-0.4$ while the corresponding measured values are $\sim 0.4-0.6$. A few explanations to this apparently less absorbing behaviour of the $\mathrm{W}$ core can be put tentatively forward. 

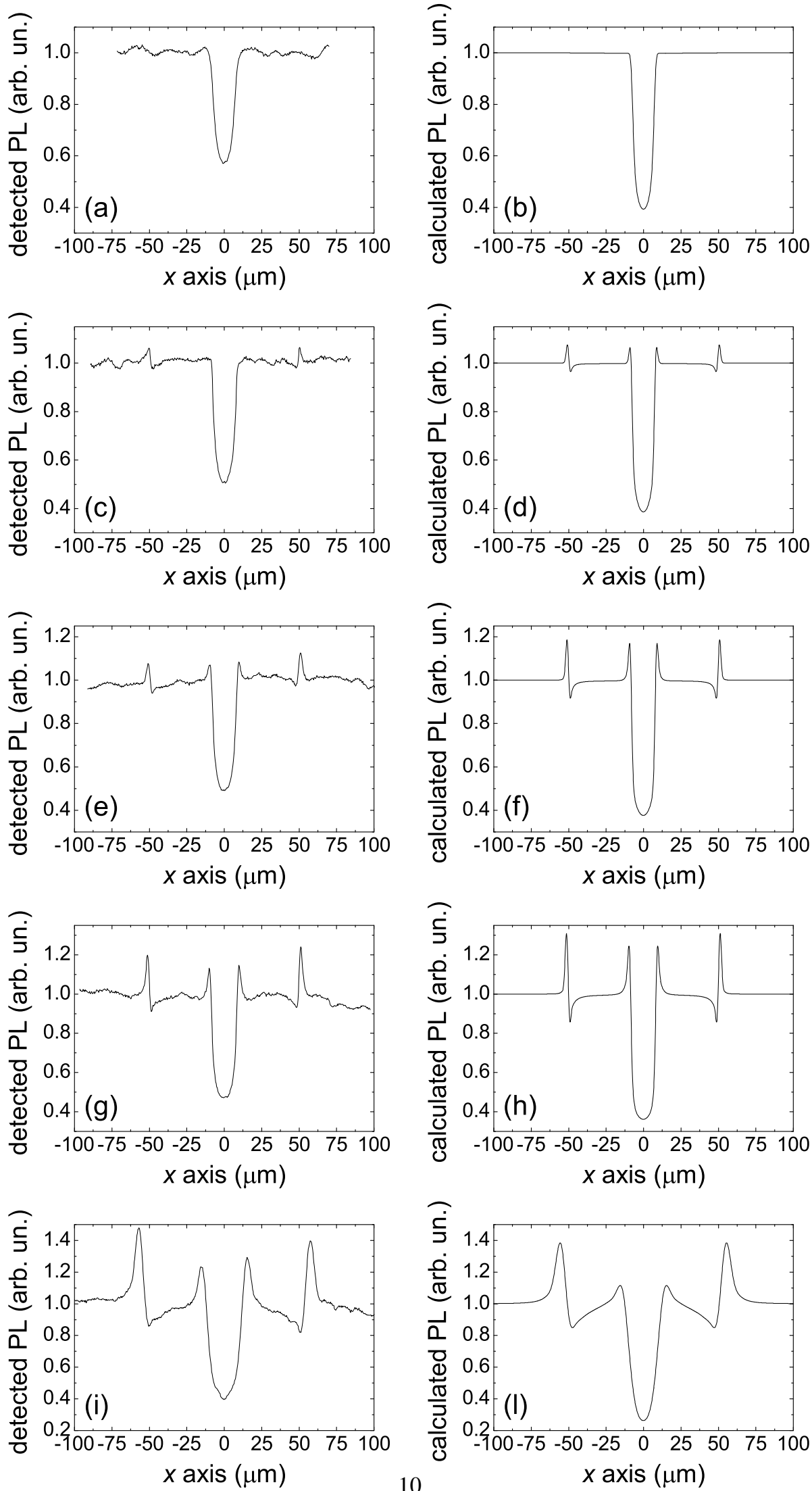

Figure 6: Measured (left-side plots) and simulated (right-side plots) PL profiles on the LiF thin-film detector placed at various distances from the BF during the irradiation stage: (a,b) $0 \mathrm{~mm}$ (contact); (c,d) $18 \mathrm{~mm}$; (e,f) $50 \mathrm{~mm}$; (g,h) $100 \mathrm{~mm}$; (i,1) $1000 \mathrm{~mm}$. The profiles on the left side are transversal scans of the PL maps shown in figure 5. 


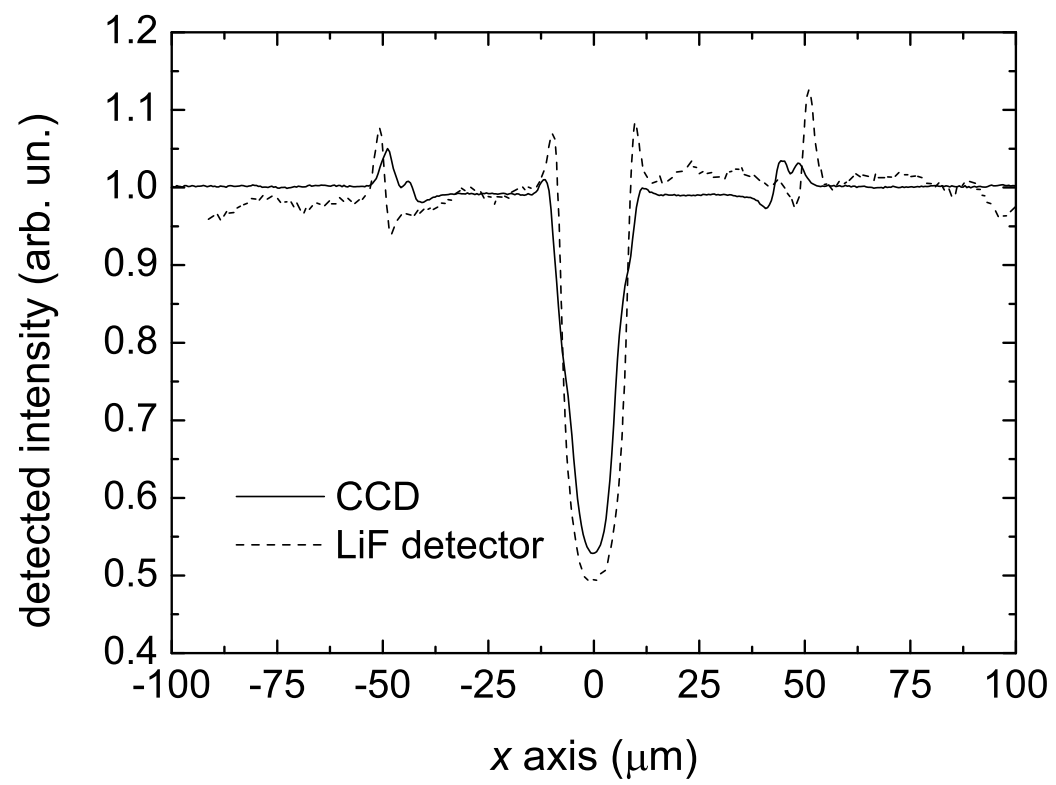

Figure 7: Comparison of the CC PL readout from the irradiated LiF film detector with an X-ray intensity-profile measurement performed by using a pco.4000 CCD camera coupled to an LSO:Tb scintillating film by a white beam microscope (Optique Peter, $10 \times$ objective, numerical aperture $\mathrm{NA}=0.14)[50,51]$. The effective pixel size of the CCD detection system was $0.45 \mu \mathrm{m}$, and the integration time was $1.5 \mathrm{~s}$. The distance between the $\mathrm{BF}$ and the detector was $50 \mathrm{~mm}$ in both cases.

1. The mass density of the $\mathrm{W}$ core could be lower than that of bulk, to which the tabulated optical constants we used correspond, because of a hypothetical lighter mass density of the wire. However, this hypothesis seems not acceptable, because the gap between measured and simulated PL intensities at the W centre would be compensated only if the actual packing density of the $\mathrm{W}$ material in the wire were $\sim 60-80 \%$, values that seem too small.

2. The response of the LiF material in terms of CC density vs. deposited energy (i.e., dose) could be nonlinear. Such an explanation has to be discarded as well, because the PL profiles measured on the LiF detectors are confirmed by similar measurements done with a CCD camera coupled to an LSO:Tb scintillating film [50, 51] — see figure 7 for a comparison at a BF-detector distance of $50 \mathrm{~mm}$-with comparable intensity values at the centre of the $\mathrm{W}$ wire shadow. Therefore, the response of the LiF detector seems to be linear, at least as far as the examined dose range is concerned.

3. The simulations contain several factors that could sum up and influence the results: a white beam, rather than a monochromatic one, is dealt with; tabulated data were used for the optical constants; simplifying hypotheses were made about the shape (Gaussian) and size of the SDF of the X-ray beam and the PSF of the CLSM objective; interference effects within the LiF film were completely disregarded. The involved multi-stage simulation process is overall a complex task and as such subjected to possible imprecisions.

4. The LiF optical constants $\delta$ and $\beta$ had to be extrapolated from tabulated data for energies higher than $30 \mathrm{keV}$ and up to $100 \mathrm{keV}$, see figure 3 . The extrapolations were based only on mathematical and graphical criteria, no further data being available at higher energies to check their reliability.

5. A stronger contribution of higher-energy photons to the generation of CC's in the LiF detector could have been overlooked. Indeed, the strong contrast between the PL corresponding to the $\mathrm{W}$ core-that is, in the spatial range $-7.5 \mu \mathrm{m} \leq x \leq 7.5 \mu \mathrm{m}$-and that of the surrounding B cladding is mostly due to the lower part of the energy spectrum. To check this fact, let us consider the energies spanned by the beam spectrum shown in figure 4: as one can verify by using the data of figure 3, the intensity transmittance of X-rays through the $\mathrm{W}$ core dramatically increases for growing $E$, while that of the B cladding is practically constant and equal to unity. In view of this, to better match our simulated PL profiles with the experimental ones of figure 6, one could assume a contribution of higher-energy photons to the formation of CC's that is to some extent larger 
than that due to lower-energy photons - in such a case a more intense PL in the W area would be obtained. Several reasons could concur to having overlooked higher-energy contributions, the most obvious ones being a possible underestimation of the imaginary part $\beta$ of the LiF refractive index at high energies (figure 3 ) or of the high-energy content of the X-ray beam spectrum (figure 4).

Explanations 4 and 5 above seem to us to be the most likely ones, also because comparison of experimental Xray intensity profiles detected by the CCD camera with corresponding PL profiles, of which an example is shown in figure 7, demonstrates the good dynamic linearity of the latter ones. Considering the complexity of the setup and of its modelling, the theoretical reproduction of the measured PL profiles in figure 6 can be recognized as a satisfactory result.

\section{Conclusions}

X-ray edge-enhanced imaging has been successfully demonstrated on LiF film detectors irradiated by a white beam in a synchrotron facility. The analyzed sample was a BF, which behaves at the operating photon energies as a mixed phase and amplitude object. It has been verified how the phase-object component of it (the cladding), because of transparency of B to X-rays, leaves no contrast on the detector when this latter is in contact with the sample (absorption-contrast technique). This fact has been confirmed by similar measurements taken with a CCD. The B cladding edges start being visible, thanks to edge enhancement, when the distance between the BF and the detector is increased. On the contrary, the $\mathrm{W}$ core wire of the $\mathrm{BF}$ is always visible, either with the BF placed in contact with or away from the detector, because of the strong X-ray absorption properties of W.

The $1.7 \mu \mathrm{m}$ thick LiF films evaporated on glass substrates have demonstrated their good usability as radiation detectors. Once irradiated, a latent image of the impinging X-ray intensity is stored in the LiF film as a spatial distribution of CC's, among which the optically active ones $\left(\mathrm{F}_{2}\right.$ and $\mathrm{F}_{3}^{+}$aggregate centres $)$photoluminesce in the visible when pumped with blue light, so that the resulting spatial distribution of PL reproduces the originally impinging X-ray intensity. The LiF film detectors provided linear response of the readout PL intensity vs. the absorbed dose-a fact confirmed by comparisons with CCD measurements (see figure 7 for one of such comparisons) —and good spatial resolution that is practically limited by the diffraction limit of the PL detection optics. In this regard, it is also worth noticing in figures 6(f) and 7 how the LiF film is apparently able to detect an intensity map which is, when compared to the CCD system, more detailed and closer in shape to the theoretical profile. Good stability even at room temperature and handling easiness make these LiF films a good alternative to other kinds of detectors.

An elementary theoretical model has been used which consists of several steps, from the interaction of the Xray beam with the sample up to the PL detection in an optical microscope. The free wave propagation of the $\mathrm{X}$ ray beam from the sample to the detector, which causes the formation of Fresnel fringes and hence of the actual edge-enhancement phenomenon, is based on the well known Fresnel's diffraction formula and has been conveniently implemented within the plane-wave spectrum formalism [43, 44]. A program for all the simulation process was written in Matlab [46]. In it, the generation of a local spatial distribution of CC's was evaluated as proportional to the deposited energy after correcting the X-ray intensity for partial spatial coherence, due to the finite size of the X-ray source, by convolution with a suitable SDF. Finally, the spatial distribution of PL radiated by the CC's in the LiF film was calculated and its detection within a CLSM was simulated by also applying a PSF correction that accounts for the instrument limited resolving power. The comparison between experimental and calculated PL profiles, shown and discussed in section 4, demonstrates the fairly good reliability of the model predictions, even though with some slight differences that are tentatively ascribed to the lack of information about the optical constants of LiF at photon energies higher than $30 \mathrm{keV}$ and/or to the underestimation of the density of higher-energy photons absorbed in LiF that contribute generating CC's.

\section{Acknowledgements}

The authors gratefully thank Massimo Piccinini for his critical reading of the manuscript. They would also like to thank the manuscript reviewers for their competent and useful comments. 


\section{References}

[1] N. W. Ashcroft, N. D. Mermin, Solid State Physics, Holt-Saunders International Editions, Philadelphia, 1981.

[2] S. Shionoya, W. M. Yen, Phosphor Handbook, CRC Press, Boca Raton, 1998

[3] W. B. Fowler, Physics of Color Centers, Academic Press, New York, 1968.

[4] T. T. Basiev, S. B. Mirov, V. V. Osiko, Room-temperature color center lasers, IEEE J. Quantum Electron. 24 (1988) 1052-1069.

[5] A. R. Lakshmanan, U. Madhusoodanan, A. Natarajan, B. S. Panigrahi, Photoluminescence of f-aggregate centers in thermal neutron irradiated LiF TLD-100 single crystals, phys. stat. sol. (a) 153 (1996) 265-273.

[6] R. M. Montereali, Point defects in thin insulating films of lithium fluoride for optical microsystems, in: H. S. Nalwa (Ed.), Ferroelectric and Dielectric Thin Films, Vol. 3 of Handbook of Thin Film Materials, Academic Press, San Diego, 2002, Ch. 7, pp. 399-431.

[7] J. Nahum, D. A. Wiegand, Optical properties of some F-aggregate centers in LiF, Phys. Rev. 154 (1967) $817-830$.

[8] J. M. Cowley, Diffraction Physics, 3rd Edition, North Holland, Amsterdam, 1995.

[9] A. Pogany, D. Gao, S. W. Wilkins, Contrast and resolution in imaging with a microfocus X-ray source, Rev. Sci. Instrum. 68 (1997) 27742782.

[10] P. Cloetens, R. Barrett, J. Baruchel, J. P. Guigay, M. Schlenker, Phase objects in synchrotron radiation hard x-ray imaging, J. Phys. D: Appl. Phys. 29 (1996) 133-146.

[11] S. W. Wilkins, T. E. Gureyev, D. Gao, A. Pogany, A. W. Stevenson, Phase-contrast imaging using polychromatic hard X-rays, Nature 384 (1996) 335-338.

[12] S. Di Fonzo, W. Jark, G. Soullié, A. Cedola, S. Lagomarsino, P. Cloetens, C. Riekel, Submicrometre resolution phase-contrast radiography with the beam from an X-ray waveguide, J. Synchrotron Rad. 5 (1998) 376-378.

[13] F. Arfelli, M. Assante, V. Bonvicini, A. Bravin, G. Cantatore, E. Castelli, L. Dalla Palma, M. Di Michiel, R. Longo, A. Olivo, S. Pani, D. Pontoni, P. Poropat, M. Prest, A. Rashevsky, G. Tromba, A. Vacchi, E. Vallazza, F. Zanconati, Low-dose phase contrast x-ray medical imaging, Phys. Med. Biol. 43 (1998) 28452852.

[14] G. Margaritondo, G. Tromba, Coherence-based edge diffraction sharpening of x-ray images: a simple model, J. Appl. Phys. 85 (1999) 3406-3408.

[15] P. Cloetens, W. Ludwig, J. Baruchel, J. P. Guigay, P. Pernot-Rejmánková, M. Salomé-Pateyron, M. Schlenker, J. Buffière, E. Maire, G. Peix, Hard X-ray phase imaging using simple propagation of a coherent synchrotron radiation beam, J. Phys. D: Appl. Phys. 32 (1999) A145-A151.

[16] Y. Suzuki, N. Yagi, K. Uesugi, X-ray refraction-enhanced imaging and a method for phase retrieval for a simple object, J. Synchrotron Rad. 9 (2002) 160-165.

[17] M. Born, E. Wolf, Principles of Optics, 6th Edition, Pergamon Press, Oxford, 1987.

[18] S. Lagomarsino, A. Cedola, X-ray microscopy and nanodiffraction, in: H. S. Nalwa (Ed.), Encyclopedia of Nanoscience and Nanotechnology, Vol. 10, American Scientific Publishers, USA, 2004, pp. 681-710.

[19] G. Baldacchini, F. Bonfigli, A. Faenov, F. Flora, R. M. Montereali, D. Murra, E. Nichelatti, T. Pikuz, Luminescent patterns based on color centers generated in lithium fluoride by extreme ultraviolet radiation and soft X-rays, Radiat. Eff. Defects Solids 157 (6-12) (2002) $569-573$.

[20] G. Baldacchini, F. Bonfigli, A. Faenov, F. Flora, R. Montereali, A. Pace, T. Pikuz, L. Reale, Lithium fluoride as a novel x-ray image detector for biological $\mu$-world capture, J. Nanosci. Nanotechnol. 3 (2003) 483-486.

[21] G. Baldacchini, S. Bollanti, F. Bonfigli, F. Flora, P. Di Lazzaro, A. Lai, T. Marolo, R. M. Montereali, D. Murra, A. Faenov, T. Pikuz, E. Nichelatti, G. Tomassetti, A. Reale, L. Reale, A. Ritucci, T. Limongi, L. Palladino, M. Francucci, S. Martellucci, G. Petrocelli, Soft X-ray submicron imaging detector based on point defects in LiF, Rev. Sci. Instrum. 76 (2005) 113104 1-12.

[22] S. Almaviva, F. Bonfigli, I. Franzini, A. Lai, R. M. Montereali, D. Pelliccia, A. Cedola, S. Lagomarsino, Hard X-ray contact microscopy with $250 \mathrm{~nm}$ spatial resolution using a LiF film detector and table-top microsource, Appl. Phys. Lett. 89 (2006) 054102 1-3.

[23] T. Kurobori, A. Matoba, Development of accurate two-dimensional dose-imaging detectors using atomic-scale color centers in Ag-activated phosphate glass and LiF thin films, Jpn. J. Appl. Phys. 53 (2014) 02BD14.

[24] S. V. Gasilov, A. Y. Faenov, T. A. Pikuz, Y. Fukuda, M. Kando, T. Kawachi, I. Y. Skobelev, H. Daido, Y. Kato, S. V. Bulanov, Wide-fieldof-view phase-contrast imaging of nanostructures with a comparatively large polychromatic soft X-ray plasma source, Opt. Lett. 34 (2009) $3268-3270$

[25] F. Bonfigli, A. Cecilia, S. Heidari Bateni, E. Nichelatti, D. Pelliccia, F. Somma, P. Vagovic, M. A. Vincenti, T. Baumbach, R. M. Montereali, In-line X-ray lensless imaging with lithium fluoride film detectors, Radiat. Meas. 56 (2013) 277-280.

[26] R. Larciprete, L. Gregoratti, M. Danailov, M. Kiskinova, R. M. Montereali, F. Bonfigli, Direct writing of fluorescent patterns on LiF films by X-ray microprobe scanning, Appl. Phys. Lett. 80 (2002) 3862-3864.

[27] S. Heidari Bateni, F. Bonfigli, A. Cecilia, T. Baumbach, D. Pelliccia, F. Somma, M. A. Vincenti, R. M. Montereali, Optical characterization of lithium fluoride detectors for broadband X-ray imaging, Nucl. Instr. Meth. A 720 (2013) 109-112.

[28] L. Reale, F. Bonfigli, A. Lai, F. Flora, A. Poma, P. Albertano, S. Bellezza, R. M. Montereali, A. Faenov, T. Pikuz, S. Almaviva, M. A. Vincenti, M. Francucci, P. Gaudio, S. Martellucci, M. Richetta, X ray microscopy of plant cells by using LiF crystal as detector, Microsc. Res. Tech. 71 (2008) 839-848.

[29] L. Reale, F. Bonfigli, A. Lai, F. Flora, P. Albertano, M. L. Di Giorgio, L. Mezi, R. M. Montereali, A. Faenov, T. Pikuz, S. Almaviva, M. Francucci, P. Gaudio, S. Martellucci, M. Richetta, A. Poma, Contact X-ray microscopy of living cells by using LiF crystal as imaging detector, J. Microsc. 258 (2015) 127-139.

[30] G. Tomassetti, A. Ritucci, A. Reale, L. Arizza, F. Flora, R. M. Montereali, A. Faenov, T. Pikuz, Two-beam interferometric encoding of photoluminescent gratings in LiF crystals by high-brightness tabletop soft x-ray laser, Appl. Phys. Lett. 85 (2004) $4163-4165$.

[31] D. Hampai, S. B. Dabagov, G. Della Ventura, F. Bellatreccia, M. Magi, F. Bonfigli, R. M. Montereali, High resolution X-ray imaging by polycapillary optics and lithium fluoride detectors combination, EPL 96 (2011) 60010 1-4.

[32] H. W. Herring, Selected mechanical and physical properties of boron filaments, Tech. rep., NASA - National Aeronautics and Space Administration, Washington, D. C. (January 1966). 
[33] C. Raven, A. Snigirev, I. Snigireva, P. Spanne, A. Souvorov, V. Kohn, Phase-contrast microtomography with coherent high-energy synchrotron x rays, Appl. Phys. Lett. 69 (1996) 1826-1828.

[34] A. Koch, C. Raven, P. Spanne, A. Snigirev, X-ray imaging with submicrometer resolution employing transparent luminescent screens, J. Opt. Soc. Am. A 15 (1998) 1940-1951.

[35] V. Kohn, I. Snigireva, A. Snigirev, Interferometric characterization of spatial coherence of high energy synchrotron X-rays, Opt. Commun. 198 (2001) 293-309.

[36] P. J. McMahon, A. G. Peele, D. Paterson, K. A. Nugent, A. Snigirev, T. Weitkamp, C. Rau, X-ray tomographic imaging of the complex refractive index, Appl. Phys. Lett. 83 (2003) 1480-1482.

[37] B. M. Song, T. A. Shelkovenko, S. A. Pikuz, M. A. Mitchell, K. M. Chandler, D. A. Hammer, X pinch X-ray radiation above 8 kev for application to high-resolution radiography of biological specimens, IEEE T. Nucl. Sci. 51 (2004) 2514-2519.

[38] O. Chubar, A. Fluerasu, Y. S. Chu, L. Berman, L. Wiegart, W.-K. Lee, J. Baltser, Experimental characterization of X-ray transverse coherence in the presence of beam transport optics, J. Phys.: Conf. Ser. 425 (2013) 052028.

[39] ANKA - the Synchrotron Radiation Facility at KIT: TOPO-TOMO webpage.

URL http: //www. anka.kit.edu/990.php

[40] The European Synchrotron, X-ray Oriented Programs (XOP) webpage. URL http://www.esrf.eu/Instrumentation/software/data-analysis/xop2.4

[41] CXRO - The Center for X-ray Optics: X-ray Database webpage. URL http://henke.lbl.gov/optical_constants

[42] R. M. Montereali, F. Bonfigli, F. Menchini, M. A. Vincenti, Optical spectroscopy and microscopy of radiation-induced light-emitting point defects in lithium fluoride crystals and films, Low Temp. Phys. 38 (2012) 779-785.

[43] J. W. Goodman, Introduction to Fourier Optics, McGraw-Hill Physical and Quantum Electronics Series, McGraw-Hill Book Company, New York, 1968.

[44] J. D. Gaskill, Linear Systems, Fourier Transforms, and Optics, John Wiley \& Sons, New York, 1978.

[45] E. O. Brigham, The Fast Fourier Transform, Prentice-Hall, Englewood Cliffs, 1974.

[46] MathWorks ${ }^{\circledR}$ MATLAB, v. 7.10.0 (R2010a), The MathWorks Inc., Natick, Massachusetts (2010). URL http: //www . mathworks . com

[47] A. Olivo, R. Speller, Experimental validation of a simple model capable of predicting the phase contrast imaging capabilities of any x-ray imaging system, Phys. Med. Biol. 51 (2006) 3015-3030.

[48] U. Platt, J. Stutz, Differential Optical Absorption Spectroscopy, Physics of Earth and Space Environments, Springer-Verlag, Berlin Heidelberg, 2008.

[49] C. S. Williams, O. A. Becklund, Introduction to the Optical Transfer Function, John Wiley \& Sons, New York, 1989.

[50] A. Rack, T. Weitkamp, S. Bauer Trabelsi, P. Modregger, A. Cecilia, T. dos Santos Rolo, T. Rack, D. Haas, R. Simon, R. Heldele, M. Schulz, B. Mayzel, A. N. Danilewsky, T. Waterstradt, W. Diete, H. Riesemeier, B. R. Müller, T. Baumbach, The micro-imaging station of the topotomo beamline at the anka synchrotron light source, Nucl. Instr. Meth. B 267 (2009) 1978-1988.

[51] A. Cecilia, V. Jary, M. Nikl, E. Mihokova, D. Hänschke, E. Hamann, P.-A. Douissard, A. Rack, T. Martin, B. Krause, M. Fiederle, T. Baumbach, Investigation of the luminescence, crystallographic and spatial resolution properties of lso:tb scintillating layers used for X-ray imaging applications, Radiat. Meas. 62 (2014) 28-34. 